\title{
A NOVEL COLOR-BASED FEATURE EXTRACTION METHOD FOR SVM BASED SKIN SEGMENTATION
}

\author{
Mehmet FIDAN 1 (iD , Utku KAYA 2 , * \\ ${ }^{1}$ Vocational School of Transportation, Eskişehir Technical University Eskişehir, Turkey \\ 2 Vocational School of Transportation, Eskișehir Technical University Eskişehir, Turkey
}

\begin{abstract}
The colored digital images can be represented in different color spaces. The most used color space is Red-Green-Blue space. However, this space can be transformed to Luminance-Blue Difference-Red Difference space for extraction of light intensity information and Hue-Saturation-Value space. The defined features of color pixels give strong information about whether they belong to a human skin or not. In this paper, a novel color-based feature extraction method is proposed, which use both red, green, blue, luminance, hue and saturation information. The proposed method is applied on an image database consists of various people with diverse age, racial and gender characteristics. The obtained features are used to segment the human skin by using Support-Vector- Machine algorithm and finally the promising performance results are presented comparatively with the most-common methods in the literature.
\end{abstract}

Keywords: Feature extraction, Image segmentation, Support Vector Machine

\section{INTRODUCTION}

The issue of skin segmentation is an important step especially in the recognition of hand and hand signs from pictures, face recognition and person tracking in videos as discussed in the work of Zarit, Super and Quek [1]. Although this work by Zarit, Super and Quek is a relatively old study of 1999, current studies on this issue show that the skin segmentation issue has not been outdated and is a suitable subject to be improved [2-9]. In addition, methods used in skin segmentation are improved and are frequently used in medical issues such as skin lesion detection [10-16].

Color histogram was used in the pioneer study for skin segmentation, where skin segmentation was used to separate hand figure from environment [17]. In another work, skin tone based on luminance component was used for skin segmentation, where skin segmentation was used for face detection [18]. In a more recent work, smoothed $2 \mathrm{~d}$ color histogram and gaussian distribution for color histogram were combined for skin segmentation [9]. Chakraborty, Buyan and Kumar proposed Dynamic Region Growing (DRG) method based on combination of local and global skin distribution models [6]. Chakraborty and Buyan also proposed a novel Adaptive Discriminative Analysis (ADA) for unsupervised skin classification in their more recent study [2]. Edge detection, Otsu segmentation and initial seed generation methods were combined by Mahmoodi and Sayedi to obtain more successful method for skin segmentation [19]. Shaik et al. compared skin segmentation accuracies of two primitive works, where one of them just only used Hue-Saturation-Value (HSV) representation and other used Luminance-Blue Difference-Red Difference (YCbCr) representation [7]. Al-Mohair, Mohamad-Saleh and Suandi applied combination of neural network and k-means clustering methods on YIQ representation of color images for skin segmentation [8].

In this study, a comprehensive image data set has been used, which was explained in Section 2.1 detailly. Red-Green-Blue-Luminance-Hue-Saturation (RGB-YHS) features have been used as predictor for making decision if the corresponding pixel is skin pixel or non-skin pixel. The detailed information for the proposed features was given in Section 2.2. The chosen features have been generated from the chosen data

*Corresponding Author: utkukaya@eskisehir.edu.tr

Received: 30.10.2020 Published: 27.11.2020 
set and the obtained features have been applied on Support-Vector-Machine with fine gaussian kernel, which was explained in Section 2.3. The comparative skin segmentation performances were presented in Section 3. Finally, the presented article was concluded by Section 4 with analysis of obtained results.

\section{MATERIALS AND METHODS}

\subsection{Schmugge Skin Image Data Set}

In this study, for train and test of the chosen Support-Vector-Machine classifier with fine gaussian kernel, "Schmugge skin image data set with ground truth" is used [20]. Schmugge, Jayaram, Shin and Tsap presented a comparative research for available studies in the literature for skin detection by listing the performances on the data set they created [21]. Schmugge skin image data set includes 846 various images with their ground truth for skin segmentation. In the original ground truth images, ' 0 ' was used to point the locations of exact skin pixels and ' 255 ' was used to point exact nonskin pixels. In the original ground truth images, there are also exist some intermediate values between 0 and 255 for the pixels that cannot be sure whether or not they belong to the skin. In this study, these intermediate values were mapped to whichever of the values between 0 and 255 and binarized ground truth images were obtained. The skin pixels labeled as 0 is assumed as positive class and nonskin pixels labeled as 255 is assumed as negative class.

300 of 846 images in Schmugge data set are landscape images, which do not contain any skin pixels. Therefore, their ground truth images consist entirely of values of ' 255 '.

21 of 846 images are community images, which contain multiple people with diverse age, racial and gender characteristics and resolutions of these community images are generally higher than other images.

171 of 354 images belong to female individuals with diverse age and racial characteristics. 167 of these 171 images contain only faces and other 4 contain additionally arm, hand, leg etc.

354 of 846 images belong to male individuals with diverse age and racial characteristics. 342 of these 354 male images contain only faces and other 12 contain additionally arm, hand, leg etc.

The detailed information about these images are listed in Table 1.

Table 1. Detailed content explanation of Schmugge skin image data set

\begin{tabular}{ccccc}
\hline Category & $\begin{array}{c}\text { Number of } \\
\text { images }\end{array}$ & Total no. of pixels & $\begin{array}{c}\text { Total no. of skin } \\
\text { pixels in } \\
\text { binarized ground } \\
\text { truth images }\end{array}$ & $\begin{array}{c}\text { Total no. of } \\
\text { nonskin pixels in } \\
\text { binarized ground } \\
\text { truth images }\end{array}$ \\
\hline Landscape & 300 & $3,862,410$ & 0 & $3,862,410$ \\
Community & 21 & $9,177,544$ & $1,816,387$ & $7,361,157$ \\
Female & 171 & $7,792,182$ & $2,001,988$ & $5,790,194$ \\
Male & 354 & $12,861,765$ & $4,463,134$ & $8,398,631$ \\
Total & 846 & $33,693,901$ & $8,281,509$ & $25,412,392$ \\
\hline
\end{tabular}

\subsection{Color Representations}

Colors are defined in different color spaces and converted into multi-dimensional digital or analog signals suitable for the purpose. The most common color spaces can be listed as Red-Green-Blue (RGB), Cyan-Magenta-Yellow-Black (CMYK), Luminance-Blue Projection-Red Projection (YUV), Luminance-Orange Blue Range- Purple Green Range (YIQ), Luminance-Blue Difference- Red Difference (YCbCr), Hue-Saturation-Value (HSV) and Hue-Saturation-Lightness (HSL). In this study, 
RGB, HSV and YCbCr representations are introduced and the proposed RGB-YHS features obtained from these representations are explained.

\subsubsection{RGB representation}

RGB is the abbreviation of "Red-Green-Blue". RGB refers to creating various colors by mixing three light tones. Unlike the subtractive model "Cyan-Magenta-Yellow-Black (CMYK)", RGB is an additive model. This representation was firstly proposed in 1855[22] and used for color photography in 1861[23] by James Clerk Maxwell. RGB representation has evolved according to the technology used in photography, television, and the latest personal computers. The RGB notation used in this study is an 8bit digital RGB notation, which uses 8 bits for each color band, so each color band has $2^{8}$ different possible shades. Since there are 3 different bands, $2^{24}$ different colors can be defined.

\subsubsection{HSV representation}

Since the mid-70s, specialists and researchers in computer graphics have focused on different color representations which would be more suitable for human perception than RGB representation. One of these studied representations is "Hue-Saturation-Value (HSV)" or in other words "Hue-Saturation-Brightness (HSB)" whose detailed explanation was given in the Hunt's 1977 article [24]. In Hunt's 1977 article, "Hue" was expressed as a perceptual chromatic term, while "Saturation" and "Value (Brightness)" were expressed as perceptual achromatic terms. In this study, HSV representation has been obtained by rgb2hsv function of Matlab, which uses mapping algorithm based on Hexcone model, defined in Smith's 1978 conference paper [25]. According to Smith's 1978 conference paper, the changing "Hue" corresponds to the transition along the color circle, a decrease in "Saturation" causes an increase in the whiteness of the color and a decrease in "Value" causes an increase in the darkness of the color.

\subsubsection{YCbCr representation}

The "Y" of $\mathrm{YCbCr}$ stands for luminance, "Cb" and "Cr" stand for blue difference and red difference respectively. YCbCr representation was derived from YUV representation by scaling and adjusting. The YUV model was developed for orientation of color broadcasting to black-and-white infrastructure [26]. $\mathrm{YCbCr}$ is a practical representation, which reveals the perceptual meaning of RGB representation. By the $\mathrm{YCbCr}$ representation, luminance can be defined in high resolution, which increases the accuracy in compressing and sub-sampling of the color images. In this study, $\mathrm{YCbCr}$ representation has been obtained by rgb2ycbcr function of Matlab, which was designed according to the definitions in Recommendation ITU-R BT.601-5 [27].

\subsubsection{Proposed RGB-YHS representation}

The RGB representation is open to improve for skin segmentation, because it is affected by shadow and light changes, causing skin pixels to be confused with nonskin pixels. In this study, all the features of RGB representation are inherited, and Saturation and Value features of HSV and Luminance feature of $\mathrm{YCbCr}$ are included. The reason for choosing the luminance( $\mathrm{Y}$ ) component of $\mathrm{YCbCr}$ instead of the Value (Brightness) component of the HSV representation is that luminance is an objective measurement and the brightness is the opposite subjective measurement, which is dependent to human perception. In fact, luminance and brightness are strongly correlated concepts, but luminance is predicted to be a healthier measurement than brightness. Because of this objective feature of Luminance, it is also preferred instead of Lightness in HSL representation, because lightness is also a subjective measurement unit based on human perception like brightness. The reason why the blue difference $(\mathrm{Cb})$ and red difference $(\mathrm{Cr})$ features are not preferred is that the information carried by these features can be calculated from the linear combination of R, G and B. Therefore, it is predicted that the classifier will also extract the information that it can obtain from $\mathrm{Cb}$ and $\mathrm{Cr}$ from Red-Green-Blue directly. 


\subsection{Support-Vector-Machine}

Support-Vector-Machine (SVM) is a classification method, which was originally proposed for two class problems [28]. SVM aims to find the best hyperplanes that distinguish data samples of one class from the data samples of the other class. In the original SVM, this separating hyperplane is assumed as linear. The best separating hyperplane for SVM obtains the largest margin between the two classes. Support vectors are samples of the classes closest to the separating hyperplane and these samples are on the border of the respective classes. The distance between the planes passing through the support vectors and parallel to the separating hyperplane determines the length of the margin.

\subsubsection{SVM with fine gaussian kernel}

The original maximum Support-Vector-Machine algorithm proposed by Vapnik in 1963 was designed as a linear classifier, as mentioned earlier. However, it was observed that it was not sufficient to classify real life data. In real life data, the hyperplane between two classes could be indented or curved, which can be defined only by a nonlinear function. In order to solve this problem, Aiserman, Braverman, Emmanuel and Rozonoer first proposed kernel trick in 1964 [29]. Bernhard Boser, Isabelle Guyon and Vladimir Vapnik improved this proposed kernel trick and applied it to SVM in 1992 [30]. The resulting nonlinear classifier was replaced by a nonlinear kernel function of each data sample. Thus, a maximum-margin hyperplane fit into a transformed feature space is achieved. Although the classifier has a linear hyper plane in the transformed feature space, it shows nonlinear property in the original sample space. The mostly used nonlinear kernel types can be listed as quadratic, cubic and gaussian. The other name of the gaussian kernel is radial basis function (RBF). The general equation of the gaussian kernel is given in (1).

$$
\mathrm{k}\left(\overrightarrow{\mathrm{x}}_{\mathrm{i}}, \overrightarrow{\mathrm{x}}_{\mathrm{j}}\right)=\mathrm{e}^{-\frac{\left\|\overrightarrow{\mathrm{x}}_{\mathrm{i}}-\overrightarrow{\mathrm{x}}_{\mathrm{j}}\right\|^{2}}{2 \sigma^{2}}}
$$

In the fine tuning of the Gaussian kernel, Matlab offers 3 different options: coarse, medium and fine. The fine gaussian kernel used in this study allows for a detailed separation of classes from each other by defining kernel scale as $\sqrt{P} / 4$, where $\mathrm{P}$ is the number of predictors.

\section{COMPARATIVE RESULTS}

As shown in Table 1, Schmugge skin image data set contains 33,693,901 number of pixels, where $8,281,509$ of them are skin pixels and 25,412,392 of them are nonskin pixels. Due to these numbers, it can be said that $24.58 \%$ of pixels belong to skin and $75.42 \%$ of pixels belong to nonskin regions, which means that the skin class (positive class) is minority class and nonskin class (negative class) is majority class. These ratios cause unbalanced classification problem.

In this study, the unbalanced classification problem is solved by training the fine gaussian SVM with equal number of randomly selected skin and nonskin pixels. 16564 random pixels whose 8282 of them belong to skin and 8282 of them nonskin pixels are chosen to train the SVM. When the pixels are randomly selected, it is guaranteed that it is absolutely unknown which images they belong to, and the chosen pixels are also shuffled among themselves so that the classifier does not memorize.

The size of train set is $0.05 \%$ of the size of entire set, which means shallow learning is applied to the SVM with fine gaussian kernel. On the other hand, the trained classifier is tested by whole set of landscape images, whole set of community images, whole set of female images, whole set of male images and finally entire Schmugge data set, respectively.

An example for a skin segmentation on a landscape image is illustrated in Figure 1. 


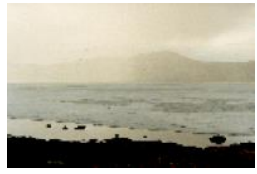

(a) (b)

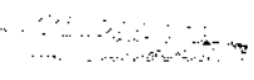

(c) (d)

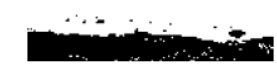

(e) (f)

Figure 1. Fine Gaussian SVM predictions for a landscape image (a) Original image (b) Ground truth

(c) Prediction by RGB features (d) Prediction by HSV features (e) Prediction by YCbCr features

(f) Prediction by RGB-YHS features

All classifiers based on RGB, HSV, YCbCr and RGB-YHS features mistakenly detected some skin pixels due to their similarity to the skin pixels in the training set. Especially, YCbCr based Fine Gaussian SVM confused for dark beach pixels in the given example. Overall performances of the fine gaussian SVM for 300 landscape images are given in Table 2.

Table 2. Performances of SVM with fine gaussian kernel for landscape images

\begin{tabular}{ccccc}
\hline Features & $\begin{array}{c}\text { Average } \\
\text { Accuracy }\end{array}$ & $\begin{array}{c}\text { Average } \\
\text { Sensitivity }\end{array}$ & $\begin{array}{c}\text { Average } \\
\text { Specificity }\end{array}$ & $\begin{array}{c}\text { Average } \\
\text { F1 score }\end{array}$ \\
\hline RGB & 0.8986 & 1 & 0.8986 & 0.0535 \\
HSV & 0.8920 & 1 & 0.8920 & 0.0535 \\
YCbCr & 0.8973 & 1 & 0.8973 & 0.0502 \\
RGB-YHS & 0.8946 & 1 & 0.8946 & 0.0500 \\
\hline
\end{tabular}

RGB-YHS features give a performance between HSV and RGB for landscape images via accuracy and specificity. The average sensitivities of all classifiers are 1 for landscape images, because there is no positive class sample to sense in landscape images. The existence of false positives causes F1 scores to converge 0 . An example for a skin segmentation on a community image is illustrated in Figure 2.

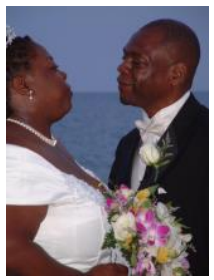

(a)

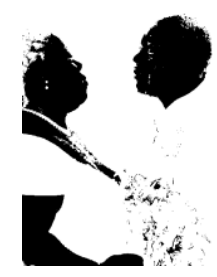

(c)

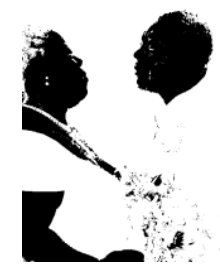

(d)

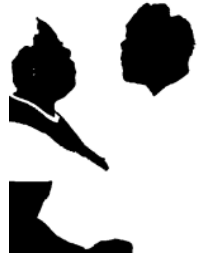

(b)

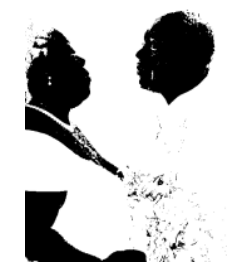

(e)

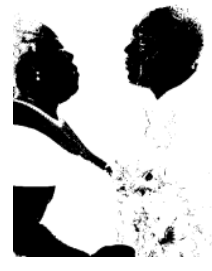

(f)

Figure 2. Fine Gaussian SVM predictions for a community image (a) Original image (b) Ground truth (c) Prediction by RGB features (d) Prediction by HSV features (e) Prediction by $\mathrm{YCbCr}$ features (f) Prediction by RGB-YHS features 
For the given sample for community image, RGB-YHS features are seen to optimize the balance between sensitivity and specificity by increasing both true positives and false negatives. The detailed performances for 21 community images are given in Table 3.

Table 3. Performances of SVM with fine gaussian kernel for community images

\begin{tabular}{ccccc}
\hline Features & $\begin{array}{c}\text { Average } \\
\text { Accuracy }\end{array}$ & $\begin{array}{c}\text { Average } \\
\text { Sensitivity }\end{array}$ & $\begin{array}{c}\text { Average } \\
\text { Specificity }\end{array}$ & $\begin{array}{c}\text { Average } \\
\text { F1 score }\end{array}$ \\
\hline RGB & 0.8575 & 0.7473 & 0.8965 & 0.6708 \\
HSV & 0.8597 & 0.7622 & 0.8947 & 0.6791 \\
YCbCr & 0.8488 & 0.7291 & 0.8892 & 0.6466 \\
RGB-YHS & 0.8551 & 0.7450 & 0.8922 & 0.6655 \\
\hline
\end{tabular}

According to Table 3, proposed RGB-YHS features show a performance between HSV and YCbCr for community images. An example for a skin segmentation on a female image is illustrated in Figure 3.

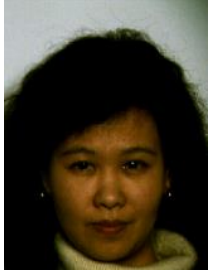

(a)

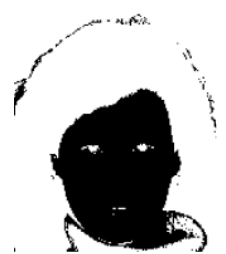

(c)

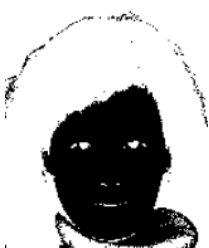

(d)

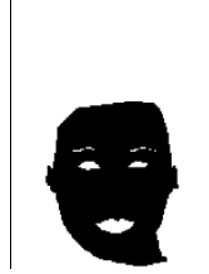

(b)

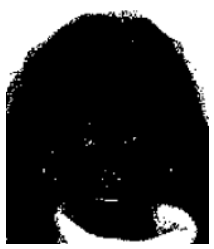

(e)

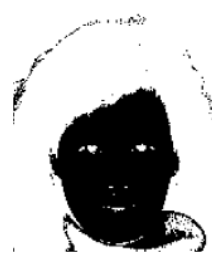

(f)

Figure 3. Fine Gaussian SVM predictions for a female individual image (a) Original image (b) Ground truth (c) Prediction by RGB features (d) Prediction by HSV features (e) Prediction by YCbCr features (f) Prediction by RGB-YHS features

The first thing to notice when looking at Figure 3 is that the fine gaussian SVM classifier, trained with YCbCr features, perceives hair as skin. The proposed RGB-YHS features decrease the false positives on lips and eyes. On the other hand, RGB-YHS cause false positives on nostrils, which can only be avoided by RGB features. The detailed performances for 171 female images are given in Table 4 .

Table 4. Performances of SVM with fine gaussian kernel for female images

\begin{tabular}{ccccc}
\hline Features & $\begin{array}{c}\text { Average } \\
\text { Accuracy }\end{array}$ & $\begin{array}{c}\text { Average } \\
\text { Sensitivity }\end{array}$ & $\begin{array}{c}\text { Average } \\
\text { Specificity }\end{array}$ & $\begin{array}{c}\text { Average } \\
\text { F1 score }\end{array}$ \\
\hline RGB & 0.8707 & 0.8906 & 0.8587 & 0.8036 \\
HSV & 0.8728 & 0.8855 & 0.8651 & 0.8054 \\
YCbCr & 0.8440 & 0.9224 & 0.8005 & 0.7943 \\
RGB-YHS & 0.8727 & 0.8928 & 0.8586 & 0.8072 \\
\hline
\end{tabular}

According to Table 4, it can be said that best F1 score is obtained by proposed RGB-YHS features, which means that RGB-YHS features optimize the balance between sensitivity and specificity. On the other hand, YCbCr gives maximum sensitivity and minimum specificity in female images. An example for a skin segmentation on a male image is illustrated in Figure 4. 


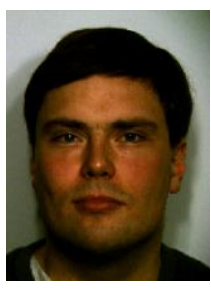

(a)

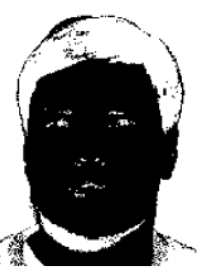

(c)

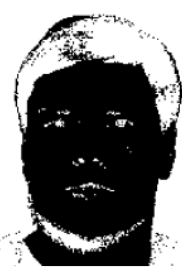

(d)

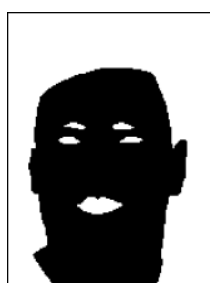

(b)

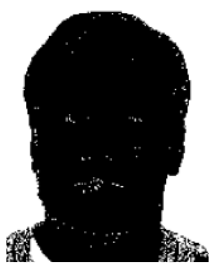

(e)

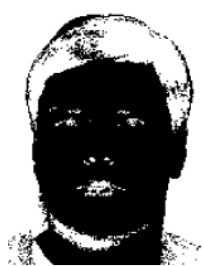

(f)

Figure 4. Fine Gaussian SVM predictions for a male individual image (a) Original image (b) Ground truth (c) Prediction by RGB features (d) Prediction by HSV features (e) Prediction by $\mathrm{YCbCr}$ features (f) Prediction by RGB-YHS features

The fine gaussian SVM classifier, trained with YCbCr features, also perceives hair as skin for male image as in the female image. The proposed RGB-YHS features decrease the false positives on lips and eyes as in the female image. The shadow under the chin and above the neck was mistakenly perceived as a nonskin region for all other features except YCbCr. The detailed performances for 354 male images are given in Table 5.

Table 5. Performances of SVM with fine gaussian kernel for male images

\begin{tabular}{ccccc}
\hline Features & $\begin{array}{c}\text { Average } \\
\text { Accuracy }\end{array}$ & $\begin{array}{c}\text { Average } \\
\text { Sensitivity }\end{array}$ & $\begin{array}{c}\text { Average } \\
\text { Specificity }\end{array}$ & $\begin{array}{c}\text { Average } \\
\text { F1 score }\end{array}$ \\
\hline RGB & 0.8676 & 0.8962 & 0.8355 & 0.8392 \\
HSV & 0.8702 & 0.8950 & 0.8427 & 0.8411 \\
YCbCr & 0.8476 & 0.9249 & 0.7748 & 0.8297 \\
RGB-YHS & 0.8681 & 0.9036 & 0.8296 & 0.8414 \\
\hline
\end{tabular}

Best F1 score is obtained also by proposed RGB-YHS features for male images, which means that RGBYHS features optimize the balance between sensitivity and specificity as in female images. On the other hand, YCbCr gives also maximum sensitivity and minimum specificity in male images. The detailed performances for all 846 images in Schmugge skin image data set are given in Table 6.

Table 6. Performances of SVM with fine gaussian kernel for entire Schmugge skin image data set

\begin{tabular}{ccccc}
\hline Features & $\begin{array}{c}\text { Average } \\
\text { Accuracy }\end{array}$ & $\begin{array}{c}\text { Average } \\
\text { Sensitivity }\end{array}$ & $\begin{array}{c}\text { Average } \\
\text { Specificity }\end{array}$ & $\begin{array}{c}\text { Average } \\
\text { F1 score }\end{array}$ \\
\hline RGB & 0.8790 & 0.9270 & 0.8641 & 0.5498 \\
HSV & 0.8782 & 0.9258 & 0.8660 & 0.5512 \\
YCbCr & 0.8645 & 0.9450 & 0.8263 & 0.5421 \\
RGB-YHS & 0.8781 & 0.9305 & 0.8601 & 0.5501 \\
\hline
\end{tabular}

According to Table 6, the proposed RGB-YHS features show similar performance with HSV in terms of accuracy. The proposed RGB-YHS show better sensitivity than RGB and HSV. The sensitivity of RGB-YHS is less than YCbCr, but the specificity of RGB-YHS is much better than YCbCr. The F1 score of RGB-YHS is better than RGB and $\mathrm{YCbCr}$. 


\section{CONCLUSIONS}

The advantage of the proposed RGB-YHS features is especially evident in male and female pictures containing only facial pixels. The success of the proposed RGB-YHS in community images was only able to surpass $\mathrm{YCbCr}$, but this problem can be solved by training the fine gaussian SVM classifier with more samples of community images in future studies. In addition, the applied shallow learning should be deepened in order to prevent the wrong skin pixel detection in the landscape pictures in future studies. It is not prevented that intense shadows on the skin cause false nonskin pixel recognition in all color representations. In this case, it is thought that color information is not sufficient. This situation is thought to be solved by a region-based study, not pixel-based study. Although region-based studies are available in the literature, the success of this proposed research can also be increased in future studies by including region-based approaches. It is thought that high performance will be achieved with deep learning methods, but due to the nature of the deep learning, it will increase the computational complexity of the training stage to very high values. By conclusion, the study conducted with the proposed method showing promising performances on the selected dataset is considered to be open to development by expanding the proposed features, improving the classifier, and using a more comprehensive training set.

\section{REFERENCES}

[1] Zarit BD, Super BJ, Quek FKH. Comparison of Five Color Models in Skin Pixel Classification. In: ICCV'99, 1999; doi: 10.1109/RATFG.1999.799224.

[2] Chakraborty BK, Bhuyan MK, Image specific discriminative feature extraction for skin segmentation. Multimedia Tools and Applications 2020; doi: 10.1007/s11042-020-08762-4.

[3] Topiwala A, Al-Zogbi L, Fleiter T, Krieger A. Adaptation and Evaluation of Deep Learning Techniques for Skin Segmentation on Novel Abdominal Dataset. In: IEEE 19th International Conference on Bioinformatics and Bioengineering (BIBE); 2019.

[4] Sadik F, Subah MR, Dastider AG, Moon SA, Ahbab SS, Fattah SA. Bangla Sign Language Recognition with Skin Segmentation and Binary Masking. In: 5th IEEE International WIE Conference on Electrical and Computer Engineering (WIECON-ECE); 2019.

[5] Hua R, Wang Y. Skin Color Detection Based Super Pixel. In: 3rd IEEE International Conference on Computer and Communications (ICCC); 2017; Chengdu, China. doi: 10.1109/CompComm.2017.8322841.

[6] Chakraborty BK, Bhuyan MK, Kumar S. Combining image and global pixel distribution model for skin colour segmentation. Pattern Recognition Letters 2017; 88: 33-40; doi: 10.1016/j.patrec.2017.01.005.

[7] Shaik KB, Ganesan P, Kalist V, Sathish BS, Jenitha JMM. Comparative Study of Skin Color Detection and Segmentation in HSV and YCbCr Color Space. Procedia Computer Science 2015; 57: 41-48; doi: 10.1016/j.procs.2015.07.362.

[8] Al-Mohair HK, Mohamad Saleh J, Suandi SA. Hybrid Human Skin Detection Using Neural Network and K-Means Clustering Technique. Applied Soft Computing 2015; 33: 337-347; doi: 10.1016/j.asoc.2015.04.046.

[9] Tan WR, Chan CS, Yogarajah P, Condell J. A Fusion Approach for Efficient Human Skin Detection. IEEE Transactions on Industrial Informatics 2012; 8(1):138-147; doi: 10.1109/tii.2011.2172451. 
[10] Goyal M, Oakley A, Bansal P, Dancey D, Yap MH. Skin Lesion Segmentation in Dermoscopic Images With Ensemble Deep Learning Methods. IEEE Access 2020; 8: 4171-4181; doi: 10.1109/access.2019.2960504.

[11] Hameed N, Shabut AM, Ghosh MK, Hossain MA. Multi-class multi-level classification algorithm for skin lesions classification using machine learning techniques. Expert Systems with Applications 2020; 141: 112961; doi: 10.1016/j.eswa.2019.112961.

[12] Alkolifi Alenezi NS. A Method Of Skin Disease Detection Using Image Processing And Machine Learning. Procedia Computer Science 2019; 163: 85-92; doi: 10.1016/j.procs.2019.12.090.

[13] Chatterjee S, Dey D, Munshi S. Integration of morphological preprocessing and fractal based feature extraction with recursive feature elimination for skin lesion types classification. Comput Methods Programs Biomed 2019; 178: 201-218; doi: 10.1016/j.cmpb.2019.06.018.

[14] Wang X, Jiang X, Ding H, Liu J. Bi-directional Dermoscopic Feature Learning and Multi-scale Consistent Decision Fusion for Skin Lesion Segmentation. IEEE transactions on image processing 2019; 29: 3039-3051; doi: 10.1109/TIP.2019.2955297.

[15] Pirnog I, Marcu I, Oprea C. Automated Segmentation of Pigmented Skin Lesions Images for Smartphone Applications. In: International Semiconductor Conference (CAS); 2019; Sinaia, Romania; doi: 10.1109/SMICND.2019.8923938.

[16] Pathan S, Prabhu KG, Siddalingaswamy PC. Techniques and algorithms for computer aided diagnosis of pigmented skin lesions-A review. Biomedical Signal Processing and Control 2018; 39: 237-262; doi: 10.1016/j.bspc.2017.07.010.

[17] Ahmad S. A usable real-time 3D hand tracker. In: Proceedings of 1994 28th Asilomar Conference on Signals, Systems and Computers; 1994; IEEE, 2: 1257-1261.

[18] Hsu RL, Abdel-Mottaleb M, Jain AK. Face detection in color images. IEEE Transactions on Pattern Analysis and Machine Intelligence 2002; 24(5): 696-706; doi: 10.1109/34.1000242.

[19] Mahmoodi MR and Sayedi SM. Leveraging spatial analysis on homogonous regions of color images for skin classification. In: 4th International Conference on Computer and Knowledge Engineering (ICCKE); 2014; Mashhad, Iran: IEEE, doi: 10.1109/ICCKE.2014.6993338.

[20] skin image Data set with ground truth. [Online]. Available: https://www.researchgate.net/publication/257620282_skin_image_Data_set_with_ground_truth

[21] Schmugge SJ, Jayaram S, Shin MC, Tsap LV. Objective evaluation of approaches of skin detection using ROC analysis. Computer vision and image understanding 2007; 108(1-2): 41-51.

[22] Maxwell JC. On the Theory of Colours in Relation to Colour-blindness. 1855.

[23] Maxwell JC. On the theory of three primary colours. 1861: Royal Institution of Great Britain.

[24] Hunt RW. The specification of colour appearance. I. Concepts and terms. Color Research \& Application 1977; 2(2):55-68.

[25] Smith AR. Color gamut transform pairs. ACM Siggraph Computer Graphics 1978; 12(3): 12-19. 
[26] Wharton W, Howorth D. Principles of television reception. 1967: Pitman.

[27] Rec. ITU-R BT.601-5. Studio Encoding Parameters of Digital Television for Standard 4:3 and Wide-screen 16:9 Aspect Ratios. (1982-1986-1990-1992-1994-1995): Section 3.5.

[28] Vapnik V. Pattern recognition using generalized portrait method. Automation and remote control $1963 ; 24: 774-780$.

[29] Aiserman M, Braverman EM, Rozonoer L. Theoretical foundations of the potential function method in pattern recognition. Avtomat. i Telemeh, 1964; 25(6): 917-936.

[30] Boser BE, Guyon IM, Vapnik VN. A training algorithm for optimal margin classifiers. In: Proceedings of the fifth annual workshop on Computational learning theory; 1992: 144-152. 